\title{
Uma breve revisão sobre a hidroxiapatita: uma biocerâmica promissora
}

\author{
A brief review on hydroxiapatite: a promising bioceramics \\ Breve resenã sobre hidroxiapatite: una biocerámica prometedora
}

Recebido: 15/12/2021 | Revisado: 22/12/2021 | Aceito: 28/12/2021 | Publicado: 06/01/2022

\author{
Érica Karine Alves de Lima \\ ORCID: https://orcid.org/0000-0003-1405-1960 \\ Universidade Federal do Piauí, Brasil \\ E-mail: ericakarinelima@gmail.com \\ Elaine Ferreira dos Santos Fernandes \\ ORCID: https://orcid.org/0000-0001-7401-9204 \\ Universidade Federal do Piauí, Brasil \\ E-mail: elainefernanddes50@ufpi.edu.br \\ Nágila Iane Pacheco \\ ORCID: https://orcid.org/0000-0002-2836-1639 \\ Centro Universitário UniFacid/Wyden, Brasil \\ Universidade Federal do Piauí, Brasil \\ E-mail: nagilaiane@hotmail.com \\ Eliana Silva Aguiar \\ ORCID: https://orcid.org/0000-0003-4193-7054 \\ Universidade Federal do Piauí, Brasil \\ E-mail: 1lianagadot@ufpi.edu.br \\ Danielle Costa Lopes \\ ORCID: https://orcid.org/0000-0002-7382-1323 \\ Centro Universitário UniFacid/Wyden, Brasil \\ E-mail: dany197_@hotmail.com \\ Luiza Aragão Paiva Pires Ferreira Mendes \\ ORCID: https://orcid.org/0000-0002-4017-6102 \\ Centro Universitário UniFacid/Wyden, Brasil \\ E-mail: luizamendes300@hotmail.com
}

\begin{abstract}
Resumo
Introdução: com o aumento dos problemas de estrutura óssea resultante de acidentes e muitos outros processos inflamatórios ou doenças ligadas ao envelhecimento humano, assim torna necessário estudos para aperfeiçoar os materiais ou criar novos materiais. Objetivo: este estudo elaborou um levantamento bibliográfico sobre a biocerâmica hidroxiapatita.Resultados e Discursão: constatou-se que esse material é constituído à base de fosfato de cálcio possuem composição análoga ao da matriz óssea, sendo promissor em diversas aplicações na área da biotecnologia, possuindo excelentes propriedades, como biocompatibilidade, osteocondutividade e bioatividade. As rotas de sintetização é um dos precursores de suas características favoráveis para as diversas áreas de aplicação científicas e tecnológicas, como liberação de fármaco, a matriz é capaz de adsorver substâncias hidrofílicas e hidrofóbicas; aplicação de implantes por conta da dimensão e da morfologia dos poros que permite a passagem de corrente sanguínea; na odontologia usada em diversas funções tais como neutralizar a hipersensibilidade dentinária, mas também para reparo da esmaltação e prevenção de cárie. Conclusão: constata-se que, HA é de grande interesse para inovações tecnológicas, trazendo avanços na bioengenharia e aperfeiçoando aplicações existentes.
\end{abstract}

Palavras-chave: Biocerâmica; Biotecnologia; Fosfato de cálcio; Hidroxiapatita.

\begin{abstract}
Introduction: with the in crease in bone structure problems resulting from accidents and many other inflammatory processes or diseases related to human aging, studies are needed to improve materials or create new materials. Objective: This study carried out a bibliographical survey on hydroxyapatite bioceramics. Results and Discussion: it was found that this material is made of calcium phosphate and has a composition similar to that of bone matrix, being promising in several applications in the area of biotechnology, having excellent properties, such as biocompatibility, osteoconductivity and bioactivity. Synthesis routes are one of the precursors of its favorable characteristics for different cientific and technological application areas, such as drug release, the matrix is capable of adsorbing hydrophilic and hydrophobic substances; application of implants due to the size and morphology of the pores that allow the passage of bloodstream; in dentistry used in various functions such asto neutralize dentinal hypersensitivity, but also to repair enamel and prevent caries. Conclusion: it appears that HA is of great interest for technological innovations, bringing advances in bioengineering and improving existing applications.
\end{abstract}

Keywords: Bioceramics; Biotechnology; Calcium phosphate; Hydroxyapatite. 


\begin{abstract}
Resumen
Introducción: com el aumento de los problemas de estructura ósea derivados de accidentes y muchos otros procesos inflamatorios o enfermedades relacionadas com El envejecimiento humano, se necesitan estudios para mejorar materiales o crear nuevos materiales. Objetivo: En este estudio se realizo um relevamiento bibliográfico sobre biocerámicas de hidroxiapatita. Resultados y Discusión: se encontró que este material está hecho de fosfato de calcio y tiene una composición similar a la de la matriz ósea, siendo prometedor en varias aplicaciones em el área de labiotecnología, teniendo excelentes propiedades, como biocompatibilidad, osteoconductividad y bioactividad. Las rutas de síntesis son uno de los precursores de sus características favorables para diferentes áreas de aplicación científica y tecnológica, como La liberación de fármacos, la matriz es capaz de adsorber sustancias hidrófilas e hidrófobas; aplicación de implantes debido al tamaño y morfología de los poros que permiten El paso del torrente sanguíneo; em odontología utilizado en diversas funciones tales comopara neutralizar la hipersensibilidad dentinaria, pero también para reparar el esmalte y prevenir la caries. Conclusión: Parece que la HA es de gran interés para lãs innovaciones tecnológicas, ya que aporta avances em bioingeniería y mejora lãs aplicaciones existentes.
\end{abstract}

Palabras clave: Biocerámicas; Biotecnología; Fosfato de calcio; Hidroxiapatita.

\title{
1. Introdução
}

A biotecnologia é uma importante área da ciência referente ao desenvolvimento de novos biomateriais que podem ser empregados parcial ou totalmente em um sistema para tratamentos, bem como, para substituir quaisquer tecidos, órgãos ou função corporal (Sousa et al., 2017). Neste sentido, os biomateriais dispõem de uma elevada pureza química para não produzir reações adversas nos tecidos vivos, aplicando os princípios da biologia e engenharia, uma vez esses materiais devem apresentar propriedades físicas e biológicas compatíveis com o organismo vivo (Abreu \& Galdino, 2017).

Nos últimos anos, com o aumento dos problemas de estrutura óssea resultante de acidentes, processos inflamatórios ou doenças ligadas ao envelhecimento humano, a medida terapeutica dos de tecidos ósseos danificados do corpo humano muitas vezes passa por procedimentos de substituição ou enxertia óssea. Sendo assim, o tratemento consiste na utilização em diferentes locais do corpo, desde estruturas dentárias a implantes ortopédicos. Esse tipo de procedimento se mostrou bastante promissor, alcançando a regeneração quase completa e reabsorção do material enxertado. No entanto, para torna-seeficaz, é necessário que o material utilizado possua propriedades físico-químicas semelhantes ao tecido ósseo (Abreu \& Galdino, 2017; Farias et al., 2020).

O osso é um material composto de matriz extracelular que consiste nos minerais, colágeno, água, proteínas não colágenas, vasos sanguíneos e lipídios que contribuem para suas funções mecânicas e metabólicas. A proporção mineral do osso é constituída por fosfato de cálcio (60\%) e também é o principal constituinte do esmalte dentário (90\%) (Silva, 2019). As cerâmicas à base de fosfato de cálcio constituem de composição análoga ao da matriz óssea e apresentam excelente biocompatibilidade, osteocondutividade e bioatividade, permitindo à proliferação celular e possibilitando o processo de regeneração óssea (Viana et al., 2020; Porsani et al., 2018).

A Hidroxiapatita (HA) é uma biocerâmica constituída por fosfato de cálcio com estrutura cristalina hexagonal que permite diversas substituições atômicas durante o seu processamento. A HA é obtida naturalmente nas estruturas ósseas ou sinteticamente por meio de processos de sintetização, como pelo método de precipitação (Souza et al., 2019), sol-gel (Ferreira, 2018), troca iônica (Silva, 2019), síntese hidrotermal (Antunes, 2018), síntese por combustão (Leite et al., 2018), pechini (Carvalho et al., 2020), etc. Devido à similaridade da HA com a estrutura química dos tecidos ósseos, esse material pode ser utilizado na odontologia, substituição de articulações, liberação de fármaco, tratamentos de traumas ortopédicos e regeneração de tecidos ósseos (Viana et al., 2020; Carvalho et al., 2020).

Nessa perspectiva, as biocerâmicas sintéticas de HA possuem propriedades promissoras, como atoxicidade, biocompatibilidade e ausência de resposta a tecidos hospedeiros. Por outro lado, os implantes podem sofrer falhas a longo prazo devido a interface material-tecido, em que, as forças de estresse são produzidas a partir da incompatibilidade do Módulo de Young entre o material e o osso. Contudo, uma forma de melhorar o comportamento mecânico é controlando o processo de 
sintetização para obter microestruturas de mesma proporção, desta maneira, esse material desperta o interesse de muitos pesquisadores com o propósito de se obter novas rotas de síntese (Canillas et al., 2017).

Diante do exposto, o objetivo deste trabalho é realizar um estudo de revisão de literatura para contribuir com a geração de conhecimento ou processos relacionados à produção de HA sintética com potencial na área biomédica. Além disso, o trabalho também visa abordar acerca de sua constituição química, identificar as possíveis rotas de fabricação e listar suas principais aplicações.

\section{Metodologia}

O presente estudo concerne de uma revisão integrativa sobre a hidroxiapatita, considerando uma biocerâmica promissora, utilizando bancos de dados online como: Google School, Scopus, Web of Science, Scientific Electronic Library Online (SCIELO). Os critérios de inclusão efetuaram-se pelo uso das palavras-chaves: Bioceramics, Biotechnology, Calcium phosphate, Hydroxyapatite, utilizando-se o descritor booleano "AND". Ressaltando, a temática de hidroxopatita de cálcio e suas modalidades de aplicação. Indexou-se estudos nos referidos bancos onlines, no período dos últimos 5 anos (2017 à 2021), levantou-se 37 artigos do Google School, 27 artigos da SCIELO, 22 artgios da Web of Science e 41 da Scopus, como demonstra-se no quadro a seguir (Tabela 1).

Utilizou-se desses, apenas 22 artigos, limitando-se os resultados em inglês, espanhol e português, excluindo artigos referentes artigos duplicados, títulos que não se enquadravam nos critérios do estudo desse trabalho, comentários e resumos de conferências. Toda pesquisa realizou-se de forma independente, os pesquisadores entrando em consenso do título a realização do estudo.

Tabela 1: Fluxograma da seleção dos artigos.

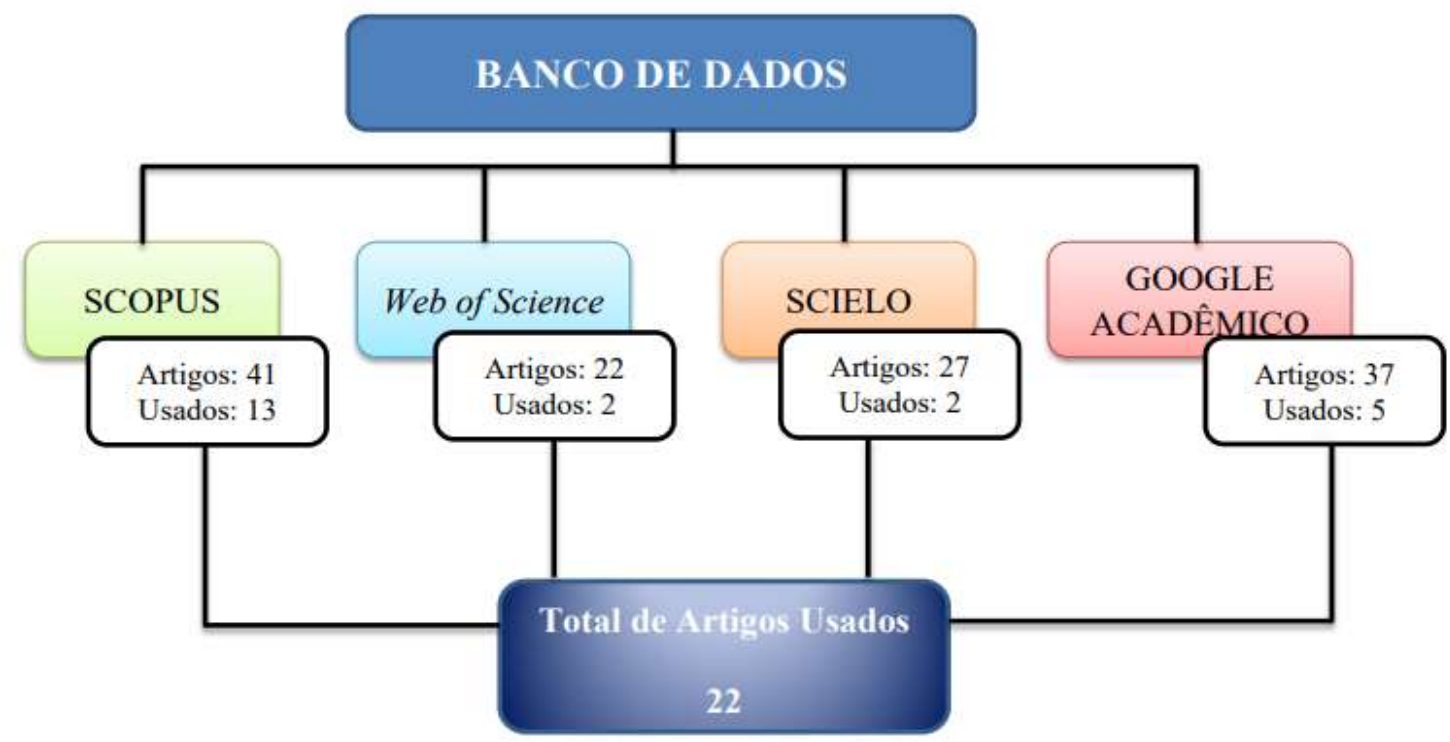

Fonte: Lima (2021).

\section{Resultados e Discussão}

\subsection{Constituição Química}

A HA é a forma mais comum do fosfato de cálcio, compreendendo a fórmula química $\mathrm{Ca}_{10}\left(\mathrm{PO}_{4}\right)_{6}(\mathrm{OH})_{2}$. Uma de suas principais propriedades é a capacidade de formar soluções sólidas e aceitar um grande número de substituintes aniônicos e 
catiônicos. A sua estrutura cristalina hexagonal pertence ao grupo espacial $\mathrm{P} 6_{3 / \mathrm{m}}$, com parâmetros de rede $\mathrm{a}=\mathrm{b}=9,43 \hat{\mathrm{A}}$ e $\mathrm{c}=$ $6,88 \AA$ A densidade de $3,16 \mathrm{~kg} / \mathrm{m}^{3}$ e razão Ca/P de 1,67, podendo ter valores aproximados de 1,5 e 2,0 (Viana et al., 2020).

A célula unitária é constituída por 10 íons de cálcio $\left(\mathrm{Ca}^{2+}\right), 6$ grupos fosfatos $\left(\mathrm{PO}_{4}{ }^{3-}\right)$ e 2 íons hidroxila $\left(\mathrm{OH}^{-}\right)$. A estrutura é composta por dois sítios de íons de cálcio não equivalentes, no qual no sítio I os íons de cálcio são organizados em colunas paralelas, enquanto que no sítio II os íons formam estruturas hexagonais, como é ilustrado na Figura 1 (Fihri et al., 2017; Viana et al., 2020).

Figura 1. Célula unitária da hidroxiapatita.

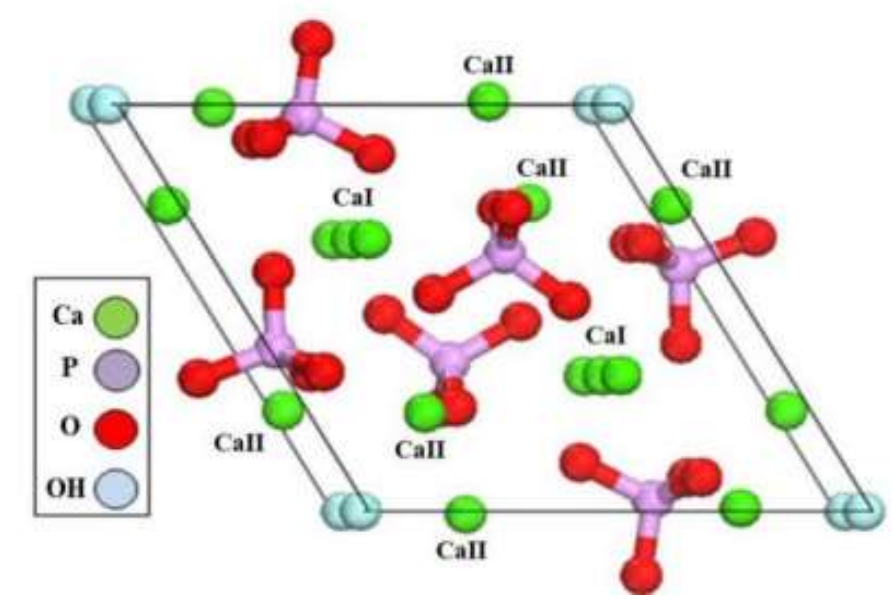

Fonte: Viana et al, (2020).

\subsection{Métodos de Síntese}

A produção de HA a baixas temperaturas resulta em um material com baixa cristalinidade, fragilidade, e a altas temperaturas, o material obtido apresenta alta cristalinidade e resistência mecânica (Viana et al., 2020).

\subsubsection{Método de Precipitação}

O método de precipitação ocorre a partir da reação do ácido fosfórico em solução com a suspensão de hidróxido de cálcio, possibilitando a formação de um produto com alto teor de pureza, obtendo HA e água, como é exibido na Equação 1 (Souza et al., 2019).

$$
10 \mathrm{Ca}(\mathrm{OH})_{2}+6 \mathrm{H}_{3} \mathrm{PO}_{4} \rightarrow \mathrm{Ca}_{10}\left(\mathrm{PO}_{4}\right)_{6}(\mathrm{OH})_{2}+18 \mathrm{H}_{2} \mathrm{O}(1)
$$

O processo geral por esse método iniciasse com a formação de uma suspensão de hidróxido de cálcio de aproximadamente $0,5 \mathrm{~mol}^{-1}$, usando hidróxido de cálcio como material de partida. a seguir, a suspensão é agitada e aquecida a $40{ }^{\circ} \mathrm{C}$ por 1 hora. Após esse período, utiliza-se uma solução de ácido fosfórico a $0,3 \mathrm{~mol} / \mathrm{L}$ com adição a uma taxa de 1 -2 gotas por segundo, sob agitação, até a formação de um precipitado em forma de gel. O precipitado é deixado em repouso por 12 horas até decantação e depois lavado, filtrado e seco em estufa a $80{ }^{\circ} \mathrm{C}$ por 12 horas. O resultado obtido é macerado e o pó é levado a um forno tipo mufla a $600{ }^{\circ} \mathrm{C}$ por 1 hora produzindo, assim, um catalisador de HA (Souza et al., 2019).

A desvantagem do método de precipitação é o controle das variáveis que influenciam as reações, como o pH (razão cálcio/fósforo) e a temperatura. $\mathrm{O} \mathrm{pH}$ é um dos fatores principais que influenciam na síntese de compostos que apresentam o grupo $\mathrm{H}_{2} \mathrm{PO}_{4}$, são obtidos em meio ácido, enquanto os grupos $\mathrm{HPO}_{4}{ }^{2-}$ e $\mathrm{PO}_{4}{ }^{3-}$, são obtidos sob condições ácidas ou alcalinas. 
Com isso, a precipitação não ocorre de forma rápida, ou seja, a titulação da solução de fosfato em solução de cálcio levar a falta de homogeneidade química no produto final (Antunes, 2018).

\subsubsection{Síntese Hidrotermal}

A síntese hidrotermal é empregada para transformar suspensões (soluções ou gel) para a fase cristalina desejada em condições leves. Essa vantagem favorece a geração de HA com alto grau de cristalinidade. Os produtos sintetizados por esse método apresentam o formato de agulha com as partículas entre 20 e $40 \mathrm{~nm}$ de diâmetro e 100-160 nm de comprimento, podendo sofrendo nucleação e adquirindo um formato esférico (Antunes, 2018).

O processo faz uso das soluções acetato de cálcio e di-hidrogenofosfato de amônio, como precursores de cálcio e fósforo, respectivamente, respeitando-se a razão estequiométrica de $\mathrm{Ca} / \mathrm{P}$. Após a mistura dos precursores de cálcio e fósforo, é adicionado o agente complexante de ácido cítrico monohidratado até a obtenção de uma solução com o pH = 4. Em seguida, acrescenta-se ureia a $0,016 \mathrm{~mol} / \mathrm{L}$. A mistura reacional é disposta em um reator e levada para a estufa por 24 horas a $180{ }^{\circ} \mathrm{C}$. Logo, realiza-se a filtração, lavagem em água destilada e secagem, obtendo partículas de HA (Antunes, 2018).

O método hidrotermal pode ser aplicado de forma convencional como já mencionado anteriormente ou fazer uso de tratamentos para a melhoria das propriedades do produto final. $\mathrm{O}$ tratamento por micro-ondas resulta em aquecimento rápido e uniforme de todo o volume da substância à temperatura de tratamento sem qualquer tensão térmica significativa ou gradiente de temperatura. Isso aumenta a cinética da reação e reduzir efetivamente a duração do processo, resultando em nucleação rápida e homogênea, onde, a síntese de HA é geralmente realizada em menos de 30 min (Antunes, 2018).

O método de processamento na parte inicial, ou seja, a preparação da mistura é o mesmo mencionado no tradicional, sendo a solução colocada em um reator e levada ao forno micro-ondas com o aquecimento gradativo até $180{ }^{\circ} \mathrm{C}$, potência de $1.000 \mathrm{~W}$ e tempo de 5 a 10 minutos. Logo após, o reator é resfriado e é realizado uma filtração simples, seguida de secagem em estufa produzindo, desta forma, as partículas de HA (Antunes, 2018).

\subsubsection{Síntese por Combustão}

O método de síntese por combustão permite a obtenção de materiais com diferentes tamanhos e morfologias, podendo obtendo as partículas de HA em escala nanométrica e melhorar, assim, a densificação (Canillas et al., 2017). O processo faz uso dos reagentes de nitrato de cálcio tetrahidratado $\mathrm{Ca}\left(\mathrm{NO}_{3}\right)_{2} \cdot 4 \mathrm{H}_{2} \mathrm{O}$ e fosfato de amônio dibásico $\left(\mathrm{NH}_{4}\right)_{2} \mathrm{HPO}_{4}$ que dissolveram-se, sepatadamente, em água destilada à temperatura ambiente, a fim de produzir soluções padrões a 2,72 e 2,09 mols/L, respectivamente. Em seguida, o combustível ureia é acrescentada à solução de nitrato de cálcio em agitação à temperatura ambiente produzindo, a primeira solução (Leite et al., 2018).

A segunda solução é o de fosfato de amônia que é adicionada a primeira solução com uma razão de gotejamento de 2 $\mathrm{ml} / \mathrm{min}$ sob constante agitação. Essas condições produzem uma solução de coloração branca com precipitados com ácido nítrico até o ponto de $\mathrm{pH}$, atinja o valor de 1. Posteriormente, a substâcia em cadinho de alumina com capacidade de $150 \mathrm{ml}$ é levada à mufla aberta, com temperaturas programadas em 500 a $800{ }^{\circ} \mathrm{C}$, para que ocorra a ignição seguida de combustão (Leite et al., 2018).

Após a combustão, a mufla é fechada por 20 minutos. As temperaturas das reações de combustão são medidas de modo online a cada 5 segundos, de acordo com a calibração do aparelho e software. Após a obtenção das amostras, os produtos das reações em forma de flocos porosos são desaglomerados em almofariz e peneirados em malha 200 (abertura $74 \mu \mathrm{m}$ ), produzindo a HA (Leite et al., 2018). 


\subsubsection{Método Pechini}

É um método sol-gel com gel polimérico orgânico bastante atrativo, entretanto, pouco discutido na literatura. Por conta disso, várias incógnitas são geradas quanto à influência das variáveis do processo de produção, dentre as quais sedestaca: a influência da relação entre o ácido cítrico/cátions metálicos. Por se tratar de um método altamente sensível, é necessário um bom controle dos parâmetros de síntese para otimizar o processo a longo prazo (Carvalho et al., 2020).

Para a síntese de nano-HA é usado água destilada em um béquer, em seguida, coloca-se esse aparato experimental em uma placa de aquecimento com agitação constante a $70{ }^{\circ} \mathrm{C}$, controlando a temperatura com o auxílio de um termômetro. $\mathrm{O}$ ácido cítrico é adicionado na solução de modo fracionado e depois, é adicionado o nitrato de cálcio e fosfato de amônio (Carvalho et al., 2020).

Após a total dissolução dos precursores (nitrato de cálcio e fosfato de amônia), o etileno glicol é incorporado na solução com proporção de 60/40 (ácido cítrico/etileno glicol). A solução é então submetida a uma temperatura entre 100 a 120 ${ }^{\circ} \mathrm{C}$ para ocorrer a reação de poliesterificação, que possui a consistência de gel. Após, ocorre a etapa de pirólise à $400{ }^{\circ} \mathrm{C}$ para a eliminação de matéria orgânica. A desaglomeração é realizada utilizando peneira ABNT n. ${ }^{\circ} 200$ seguido de calcinação na temperatura de $1000{ }^{\circ} \mathrm{C}$ resultando, deste modo, em nanopartículas de HA (Carvalho et al., 2020).

\subsubsection{Método de Troca Iônica}

O método consiste, na liberação e troca de íons presentes na superfície da HA por íons presentes em meio aquoso através da adsorção dessas espécies iônicas que, posteriormente, são expandidos para o interior da rede sem a formação de novas fases. No entanto, a possibilidade de substituir os grupos iônicos da HA por íons bifuncionais via troca iônica, evitaria problemas de transformações de fases em altas temperaturas. Os mecanismos podem ocorrer por troca iônica e dissoluçãoprecipitação, sendo que em ambos os casos acontecemna superfície da HA (Silva, 2019).

Nos processos são trocados por íons presentes na solução, que podem fazem parte da constituição dos cristais de HA sem que ocorra a formação de novas fases. O processo difusivo e de homogeneização da espécie envolvida no bulk dos cristais é facilitado para o caso de cristais de tamanho reduzido (nanocristais) ou de baixa cristalinidade. Majoritariamente, os processos ocorrem devido à presença de uma camada hidratada na superfície da HA, no qual, os íons da estrutura encontram-se fracamente ligados por interações eletrostáticas que são facilmente trocados com outros íons presentes na solução (Silva, 2019).

Os íons substituintes envolvidos no processo são incorporados nos sítios de $\mathrm{Ca}^{2+}$ da fase $\mathrm{HA}$, sendo apenas adsorvidos fisicamente na superfície. Para serem facilmente incorporados na rede da HA, os cátions substituintes envolvidos na troca iônica apresentam raios iônicos semelhantes ao $\mathrm{Ca}^{2+}$ e valores de eletronegatividade próximos ou maiores que a do íon $\mathrm{Ca}^{2+}$ (Silva, 2019).

\subsubsection{Método Sol-Gel}

Esse método é caracterizado por duas etapas, em que, uma é o "sol" que é a solução de compostos metálicos ou suspensão de partículas muito finas em um líquido, sendo transformado em uma massa altamente viscosa, o "gel". Para a síntese cerâmica são identificadas duas rotas diferentes, a depender da estrutura do gel, a saber: gel particulado ou gel polimérico (A estrutura mais relatada é a do tipo gel polimérico) (Ferreira, 2018).

O material consiste de uma solução de metais alcóxidos em um álcool apropriado. Nessa, é incorporado água no estado puro ou diluída em álcool. As reações de hidrólise e condensação podem ocorrem sob agitação constante em temperaturas maiores do que a temperatura ambiente, bem como, a concentração adequada de reagentes e do pH da solução, 
levando à formação de cadeias poliméricas. Em seguida, é realizada a calcinação entre 300 a $700{ }^{\circ} \mathrm{C}$ produzindo, desta forma, a HA, como é ilustrado na Figura 2 (Ferreira, 2018).

Figura 2. Método de síntese por sol-gel.

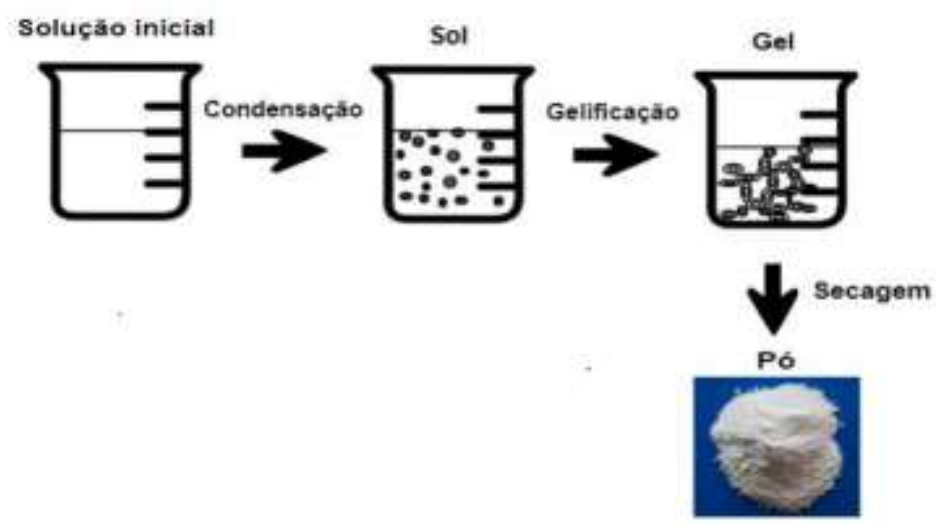

Fonte: Ferreira (2018).

Ao contrário dos outros métodos, o sol-gel utilizado para pós cerâmicos possibilita a produção de composições homogêneas a temperaturas baixas e os seus reagentes estão disponíveis com alta pureza, permitindo a obtenção de pós finos (Ferreira, 2018).

\subsection{Aplicações da Hidroxiapatita}

A HA dispõe de um alto potencial para aplicações biomédicas, tornando-se bastante utilizada na área ortopédica, como material para implantes ósseo e próteses. No entanto, também são aplicadas na liberação controlada de drogas, no revestimento de implantes e no enxertos ósseo, bem como, na confecção de andaimes para a Engenharia de Tecidos (Gomes et al., 2019). Dependendo do tipo de produto final desejado, a escolha da rota de síntese é importante para o aprimoramento das características da HA resultante. Por exemplo, a síntese através da troca iônica dificulta a homogeneização dos íons na estrutura (trata-se de um processo difusivo superfície-bulk), limitando a sua concentração (Silva, 2019).

Referindo-se a respostas biológicas, a HA produzida pelo método de troca iônica é viável para no reparo de ósseo de curto prazo, pois os íons dopantes permanecem nas camadas mais superficiais, mas são liberadas rapidamente no meio. No caso de precipitação aquosa, a resposta é mais duradora, pois apresenta uma interação maior com o tecido, logo os íons presentes substituir desde a superfície até o bulk, assim uma liberação progressiva de íons para o meio biológico (Silva, 2019).

\subsubsection{Liberação de Fármaco}

O sistema de entrega de drogas na última década tem tido um grande interesse na aplicação de HA, podendo ser funcionalizada devido à incorporação de diversos íons, possibilitando sua vetorização para locais específicos. A matriz é capaz de adsorver substâncias hidrofílicas e hidrofóbicas, sendo uma característica de grande relevância para sua aplicação como veículo de fármacos, dado os diversos tipos de composições químicas existentes (Barbosa, 2020).

A maior especificidade da HA para células neoplásicas, preferencialmente, células sadias, tornando de grande interesse na entrega de medicamentos para o câncer. Neste sentido, essas características são atribuídas por conta da maior atração eletrostática entre os sítios positivos dos cristais de HA com a elevada carga negativa de superfície em células cancerosas. A carga superficial negativa em células neoplásicas trata-se de uma particularidade que não é observada em células 
normais, que ocorrem porque as células cancerígenas metabolizam a glicose de uma forma diferente das células sadias (Barbosa, 2020).

\subsubsection{Implantes}

A superfície da HA permite a interação das ligações do tipo dipolo, possibilitando que as moléculas da água, proteínas e colágeno, sejam absorvidas na superfície e, como resultado, tem-se a regeneração tecidual. A dimensão e a morfologia dos poros são fatores cruciais para uma excelente osteointegração, e o tamanho mínimo necessário para permitir o crescimento do osso e a passagem de corrente sanguínea é de aproximadamente 100 a $150 \mu \mathrm{m}$. Entretanto, alguns autores relataram que para a excelente colonização de osteoblastos e crescimento fibroso-muscular, o tamanho dos poros está entre 200 a $500 \mu \mathrm{m}$. Outros requisitos importantes para os implantes são a interconectividade dos poros para a penetração das células semelhantes a osteoblastos em seu interior, bem como, a rugosidade superficial para a fixação das células (Freitas, 2020).

As características físicas obtidas no desenvolvimento de biocerâmicas para substitutos ósseos dependem do volume de poros, do tamanho médio dos poros e das interconexões. Um desenvolvimento bem-sucedido de substitutos ósseos com propriedades ideais requer o controle perfeito desses parâmetros. A grande diversidade de requisitos de reconstrução clínica para os defeitos do esqueleto levou ao desenvolvimento de vários métodos para preparar implantes. Isso permite a produção de HA com porosidade controlada, boa interconectividade porosa, resistência mecânica e propriedades de superfície (Freitas, 2020).

\subsubsection{Odontologia}

A HA devido às suas propriedades bioativas e sua capacidade de induzir o processo de mineralização, é utilizada com o intuito de neutralizar a hipersensibilidade dentinária. É considerado que a circulação de fluido dentro dos túbulosproduz uma excitação das células receptoras na polpa quando os túbulos dentinários são expostos. A HA pode facilmente penetrar nos túbulos dentinários e reagir como um agente mineralizante (Bordea et al., 2020; Baglar et al., 2018; Jeevanandam et al., 2018).

Alguns defeitos microscópicos de superfície e poros no esmalte permite que o agente clareador penetre e produza sensibilidade. A pasta que contêm sua composição com HA repara esses pequenos defeitos no esmalte evitando, assim, a reação sensorial (Kutuket al., 2018). Outra área em que a HA abrangem ganhado destaque é na prevenção de cárie. Quando a cárie se encontra no estágio inicial, o tecido duro perde os íons minerais por ataque de ácido proveniente de bactérias, mas a rede de colágeno permanece não afetada (Bordea et al., 2020; Kutuk et al., 2018; Gemelli et al., 2020).

Na prevenção da cárie, a HA é adicionada em pastas de dente para fornecer os íons que reduzem a desmineralização e melhorar a remineralização. As nanopartículas penetramnas porosidades dos dentes que produz uma camada protetora superficial (Anand et al., 2017).

\section{Considerações Finais}

A HA está ganhando grande destaque por suas características estruturais, tornando um material promissor possível de ser produzido por diferentes rotas de sintetização, sendo que cada rota apresenta as suas características inerentes. Observou-se que as principais rotas empregadas produzem HA em escala nanométrica, com características de alta porosidade e maior área de superfície. Essas características tornam à HA um material cerâmico atraente na área biomédica, devido a sua similaridade com a estrutura mineral dos ossos, bem como, biocompatibilidade, atoxicidade, osteocondutividade, bioatividade, etc. Portanto, acredita-se que a HA é de grande interesse para inovações tecnológicas, trazendo avanços na bioengenharia e aperfeiçoando aplicações existentes. Dessa forma, esse artigo fornece bases para novas pesquisas. 


\section{Referências}

Abreu, M. S., \& Galdino, A. G. S. (2017). Caracterização microestrutural de compósitos de hidroxiapatita-alumina confeccionados pelo método da esponja polimérica. Ceramica, 63(365), 34-38. https://doi.org/10.1590/0366-69132017633652027

Anand, S., Rejula, F., Sam, J. V. G., Christaline, R., Nair, M. G., \& Dinakaran, S. (2017). Comparative Evaluation of Effect of Nano-hydroxyapatite and 8\% Arginine Containing Toothpastes in Managing Dentin Hypersensitivity: Double Blind Randomized Clinical Trial. Acta Medica (Hradec Králové), 60(3), 114119. https://doi.org/10.14712/18059694.2018.3

Antunes, L. (2018). Síntese de hidroxiapatita pelo método hidrotermal assistido por micro-ondas. (Dissertação de mestrado, Universidade Estadual de Ponta Grossa, Ponta Grossa). Recuperado de https://tede2.uepg.br/jspui/bitstream/prefix/2604/1/LIZIANE\%20ANTUNES.pdf

Barbosa, A. A. (2020). Hidroxiapatita Multifuncional: da Síntese e Caracterização à Aplicação Biomédica. (Tese de doutorado, Universidade Federal de Pernambuco, Recife). Recuperado de https://repositorio.ufpe.br/bitstream/123456789/37063/1/TESE Amanda Alves Barbosa.pdf

Baglar, S., Erdem, U., Dogan, M., \& Turkoz, M. (2018). Dentinal tubule occluding capability of nano-hydroxyapatite; The in-vitro evaluation. Microscopy Research and Technique, 81(8), 843-854. https://doi.org/10.1002/jemt.23046

Bordea, I. R., Candrea, S., Alexescu, G. T., Bran, S., Băciuț, M., Băciuț, G., Lucaciu, O., Dinu, C. M., \& Todea, D. A. (2020). Nano-hydroxyapatite use in dentistry: a systematic review. Drug Metabolism Reviews, 52(2), 319-332. https://doi.org/10.1080/03602532.2020.1758713

Canillas, M., Rivero, R., García-Carrodeguas, R., Barba, F., \& Rodríguez, M. A. (2017). Processing of hydroxyapatite obtained by combustion synthesis. Boletín de La Sociedad Española de Cerámica y Vidrio, 56(5), 237-242. https://doi.org/10.1016/j.bsecv.2017.05.002

Carvalho, G. K. G., Almeida, Y. B. A. Alves, K. E. S., Farias, J. R. S., Simões, V. N., \& Braga, A. N. S. (2020). Avaliação Estrutural E Morfológica Da Hidroxiapatita Sintetizada Por Pechini/Structural and Morphological Evaluation of Hydroxyapatite Synthesized By Pechini. Brazilian Journal of Development, 6(9), 71812-71821. https://doi.org/10.34117/bjdv6n9-576

De Santana Souza, T., Rangel, F. C., Tokumoto, M. S., Lôbo, I. P., \& Da Cruz, R. S. (2019). Synthesis, characterization and modification of hydroxyapatite with zinc for application in the esterification reaction. Revista Materia, 24(1). https://doi.org/10.1590/s1517-707620190001.0659

De Sousa, W. J. B., Barbosa, R. C., Fook, M. V. L., Filgueira, P. T. D., \& Tomaz, A. F. (2017). Membranas de polihidroxibutirato com hidroxiapatita para utilização como biomaterial. Revista Materia, 22(4). https://doi.org/10.1590/S1517-707620170004.0236

Farias, J. R. S., Carvalho, G. K. G., \& Braga, A. N. S. (2020). Cerâmicas de fosfatos de cálcio bifásicas : uma revisão: Uma Revisão. Revista Eletrônica de Materiais e Processos, 15(2), 54-70. http://www2.ufcg.edu.br/revista-remap/index.php/REMAP/article/view/743/510.

Ferreira, C. R. D. (2018). Compósito de zircônia comercial com hidroxiapatita pura produzida via método sol gel para aplicações biomédicas. (Dissertação de mestrado, Universidade Federal do Rio Nrande Noral, https://repositorio.ufrn.br/jspui/bitstream/123456789/25486/1/Comp\%C3\%B3sitoZirc\%C3\%B4niaComercial_Ferreira_2018.pdf

Fihri, A., Len, C., Varma, R. S., \& Solhy, A. (2017). Hydroxyapatite: A review of syntheses, structure and applications in heterogeneous catalysis. Coordination Chemistry Reviews, 347(1), 48-76. https://doi.org/10.1016/j.ccr.2017.06.009

Freitas, L. A. de. (2020). Síntese e caracterização de hidroxiapatita porosa com propriedades osteoativas. (Dissertação de mestrado, Universidade Tecnológica Federal do Paraná, hondrina). ht//repositorio.utfpr.edu.br/jspui/bitstream/1/5226/1/LD_PPGCEM _M_Freitas\%2C_Lucas_Augusto_de_2020.pdf

Gemelli, E., Franco, C. J., \& Camargo, N. H. A. (2020). Elaboração de hidroxiapatita sinterizada a alta temperatura para aplicações odontológicas. Revista Materia, 25(4). https://doi.org/10.1590/s1517-707620200004.1142

Gomes, D. S., Santos, A. M. C., Neves, G. A., \& Menezes, R. R. (2019). A brief review on hydroxyapatite production and use in biomedicine. Cerâmica, 65(374), 282-302. https://doi.org/10.1590/0366-69132019653742706

Jeevanandam, J., Barhoum, A., Chan, Y. S., Dufresne, A., \& Danquah, M. K. (2018). Review on nanoparticles and nanostructured materials: History, sources, toxicity and regulations. Beilstein Journal of Nanotechnology, 9(1), 1050-1074. https://doi.org/10.3762/bjnano.9.98

Kutuk, Z. B., Ergin, E., Cakir, F. Y., \& Gurgan, S. (2019). Effects of in-office bleaching agent combined with different desensitizing agents on enamel. Journal of Applied Oral Science, 27, 1-10. https://doi.org/10.1590/1678-7757-2018-0233

Leite, R. B., Araújo, P. M. A. G., Batista, H. A., Santos, P. T. A., Bezerra, D. C., \& Costa, A. C. F. M. (2018). Avaliação da Temperatura de Aquecimento Externo na Síntese por Reação de Combustão para Obtenção da Hidroxiapatita. Revista Eletrônica de Materiais e Processos, 13(2), 119-126. http://www2.ufcg.edu.br/revista-remap/index.php/REMAP/article/view/669/452.

Porsani, N. K., Trombini, V., Ana, P. A., \& Setz, L. F. G. (2018). Avaliação reológica da hidroxiapatita. Cerâmica, 64(371), 325-330. https://doi.org/10.1590/0366-69132018643712334

Silva, C. M. P. da. (2019). Obtenção de biocimentos de hidroxiapatita e $\beta$-TCP para aplicações ortopédicas e odontológicas. (Trabalho de conclusão de curso, Universidade Federal do Ceará, Fortaleza). Recuperado de http://www.repositorio.ufc.br/bitstream/riufc/48630/1/2019_tcc_cmpsilva.pdf

Viana, J. R., Macêdo, A. A. M., Santos, A. O. Dos, Filho, P. de F. F., Graça, M. P. F., Valente, M. A., \& Silva, C. C. Da. (2020). Comparative analysis of solid state hydroxyapatite synthesis. Revista Materia, 25(1). https://doi.org/10.1590/s1517-707620200001.0914 